\title{
Error estimates derived from the data for least-squares spline fitting
}

\author{
Jerome Blair \\ National Security Technologies, LLC \\ P.O. Box 98521, Las Vegas, NV 89193-8521, MS/NLV-071 \\ Phone: +1-702-295-2647, Fax: +1-702-295-3305, Email: j.blair@ieee.org
}

\begin{abstract}
The use of least-squares fitting by cubic splines for the purpose of noise reduction in measured data is studied. Splines with variable mesh size are considered. The error, the difference between the input signal and its estimate, is divided into two sources: the $\boldsymbol{R}$-error, which depends only on the noise and increases with decreasing mesh size, and the $F$ error, which depends only on the signal and decreases with decreasing mesh size. The estimation of both errors as a function of time is demonstrated. The $R$ error estimation requires knowledge of the statistics of the noise and uses well-known methods. The primary contribution of the paper is a method for estimating the $F$-error that requires no prior knowledge of the signal except that it has four derivatives. It is calculated from the difference between two different spline fits to the data and is illustrated with Monte Carlo simulations and with an example.
\end{abstract}

Keywords - data smoothing, multiresolution analysis, spline functions, estimation

\section{INTRODUCTION}

This is the first in a series of papers on a particular class of practical methods for extracting an accurate estimate of a signal from noisy measurements. The problem, in the simplest form that will be considered, is that a signal $s(t)$ is measured at uniformly-spaced discrete times, $t_{i}$, for $i=1$ to $N$. The measurements have random noise with known statistics. Throughout this paper, it will be assumed that the measurement noise is white. However, for a few years, the author been successfully using these methods for problems in which the noise is not white and not even stationary, and the sampling very non-uniform. This problem was first systematically studied in its modern form in [1]-[3], though closely related problems were studied by Gauss [4] as far back as 1804. The measured signal is represented as $\boldsymbol{y}=\boldsymbol{s}+\boldsymbol{e}$, where $\boldsymbol{s}$ is the true signal and $\boldsymbol{e}$ is the vector of measurement errors. We estimate the signal with $\hat{s}$, where

$$
\hat{\boldsymbol{s}}=\boldsymbol{P y}=\boldsymbol{P s}+\boldsymbol{P e},
$$

where $\boldsymbol{P}$ is a linear operator that is applied to the data to give an accurate estimate of the signal. The operator, $\boldsymbol{P}$, is designed to smooth, or filter, the data to reduce the noise while not distorting the signal too much. The error in the recovered signal is given by $\boldsymbol{e}_{\boldsymbol{s}}$ with

$$
e_{s}=s-\hat{s}=s-P s-P e=(I-P) s-P e,
$$

where $\boldsymbol{I}$ is the identity operator. In Equation (2), there are two sources of error, one resulting from the term $(\boldsymbol{I}-\boldsymbol{P}) \boldsymbol{s}$ and one resulting for the term $\boldsymbol{P e}$. In this paper, the first term is called the $F$-error (which could equally mean fitting error or filtering error). It is the error the smoothing operation introduces in the absence of measurement errors. The second term is called the $R$-error, which is the error in the reconstructed signal caused by the measurement errors. Since it is assumed that there is a known statistical distribution for $\boldsymbol{e}$, the statistical distribution for $\boldsymbol{P e}$, the $R$ error, can be calculated. The new results in this paper deal with estimating the $F$-error.

In [1]-[3] and hundreds of papers and textbooks written since then, a statistical distribution is assumed known for $\boldsymbol{s}$, thus allowing the computation of a statistical distribution for $(\boldsymbol{I}-\boldsymbol{P}) \boldsymbol{s}$, the $F$-error. In this situation it is possible, for any fixed $\boldsymbol{P}$, to calculate the statistical distribution for $\boldsymbol{e}_{\boldsymbol{s}}$ and select an optimum $\boldsymbol{P}$ using the minimum mean squared error criterion. The solution to this problem is well known (c.f. Chapter 12 of [5]).

This paper will show how to estimate statistical properties of the $F$-error without any a priori statistical knowledge about the signal. Of course, some knowledge about the signal must be assumed. It is assumed that the sampling rate is more than adequate to represent the signal. Implicit in some of our calculations is that the sampling rate is a factor of five more than the minimum necessary. This assumption is relevant to practical problems, because in recent years the sampling rate and bandwidth of digital oscilloscopes has been increasing rapidly, but the noise level has remained constant or deteriorated. This makes the situation of high sampling rate and high noise level one of importance. It is also assumed that the unknown signal has four derivatives, but no assumptions are made about the magnitudes of the derivatives.

\section{SPLINE FUNCTIONS}

The smoothing operators used are based on cubic spline functions. Let the interval over which the signal is measured be $T_{1} \leq t \leq T_{2}$, and let $K$ be a sequence of time values, $t_{k}$, for $k=1$ to $n<N$ satisfying $t_{1}=T_{1}, t_{k+1}>t_{k}$ and 
$t_{n}=T_{2}$. A cubic spline with knots, $K$, is a function defined on the interval $\left[T_{1}, T_{2}\right]$ that is a polynomial of degree three or less on each sub-interval of the form $\left[t_{k}, t_{k+1}\right]$ and that has two continuous derivatives throughout the interval $\left[T_{1}, T_{2}\right]$. The intervals, $\left[t_{k}, t_{k+1}\right]$, will be called the knot intervals, and the length, $\Delta_{k}=t_{k+1}-t_{k}$, is called the mesh size (of the $k^{\text {th }}$ knot interval). The symbol, $S_{K}$, denotes the vector space of cubic spline functions with knot sequence $K$. Because of the continuity requirement on the second derivatives, the dimension of $S_{K}$ is $n+2$. These functions and many algorithms for dealing with them are described in [6]. The algorithms in [6] are given in FORTRAN. The author used the MATLAB implementation of these algorithms [7]. Differences between the FORTRAN and MATLAB implementations are discussed in [6].

\section{CALCULATING THE APPROXIMATION AND ESTIMATING THE $R$-ERROR}

This section begins with some notation, some of which has already been used in the introduction. The unknown signal is expressed by the vector, $\boldsymbol{s}$, with components $s_{i}$, which are the values of the signal at the times $t_{i}$ at which the signal is sampled. The number of samples is denoted by $N$. It is assumed that the sampling rate is more than adequate to represent the signal. The error in the measured value of the signal is expressed by the vector, $\boldsymbol{e}$, with components, $e_{i}$. The error components, $e_{i}$, are assumed to be independent, identically distributed random variables with zero mean, which implies that the error sequence is stationary white noise. The measured data is given by the vector $\boldsymbol{y}=\boldsymbol{s}+\boldsymbol{e}$.

The vector space in which all of the signals, the errors, and the measured data lie will be denoted by $\mathcal{V}$. When using matrix notation, the signal vectors are assumed to be column vectors. We define an inner product on $\mathcal{V}$ by

$$
\langle\boldsymbol{u}, \boldsymbol{v}\rangle=\Delta t \sum_{i=1}^{N} u_{i} v_{i}=\Delta t \boldsymbol{u}^{T} \boldsymbol{v},
$$

where $\Delta t$ is the inverse of the sampling frequency. The superscript, $T$, applied to any matrix gives the transpose of the matrix. In the case of a column vector this is the row vector with the same components. The multiplier, $\Delta t$, is applied so that the value of the inner product is approximately independent of the sampling frequency, being an approximation of the integral inner product for continuous-time signals. Corresponding to the inner product is the norm given by

$$
\|\boldsymbol{u}\|=\sqrt{\langle\boldsymbol{u}, \boldsymbol{u}\rangle} .
$$

For a specified sequence of knots, $K$, as described in the introduction, the set of cubic splines with the specified knots will be denoted by $\mathcal{S}_{K}$. The signal estimate, $\hat{\boldsymbol{s}}$, is given by $\hat{\boldsymbol{s}}$ is the element of $\mathcal{S}_{K}$ which minimizes $\|\hat{\boldsymbol{s}}-\boldsymbol{y}\|^{2}$.

This is the least squares estimate by splines that is covered in the second half of Chapter XIV of [6]. It is shown there that

$$
\hat{\boldsymbol{s}}=\boldsymbol{P y},
$$

where $\boldsymbol{P}$ is a linear operator. We will use the symbol, $\boldsymbol{P}$, to represent both the linear operator and the matrix that represents it. It is also shown in the reference that

$$
\boldsymbol{P}^{T}=\boldsymbol{P} \text { and } \boldsymbol{P}^{2}=\boldsymbol{P} .
$$

These are properties of least-squares fitting operators in general and have nothing specific to do with spline fitting.

Although this operator formalism is useful for deriving properties of and results about spline fitting, it is not customary to actually calculate the $\boldsymbol{P}$ matrix in the course of the calculations. There are programs available in the references that take $K$ and $\boldsymbol{y}$ as input and calculate $\boldsymbol{P} \boldsymbol{y}$ as an output without ever calculating $\boldsymbol{P}$. In situations in which more than one knot sequence is under consideration, the knot sequence name may be used as a subscript on both $\boldsymbol{P}$ and $\hat{\boldsymbol{s}}$.

It is of interest to determine how the value of $\hat{\boldsymbol{s}}$ at any particular time depends on the data, $\boldsymbol{y}$. To this end let $\boldsymbol{\delta}_{j}$ be the vector whose $j^{\text {th }}$ component is $1 / \Delta t$ while all other components are zero. We then have

$\hat{s}_{j}=\left\langle\boldsymbol{\delta}_{j}, \hat{\boldsymbol{s}}\right\rangle=\left\langle\boldsymbol{\delta}_{j}, \boldsymbol{P} \boldsymbol{y}\right\rangle=\left\langle\boldsymbol{P}^{T} \boldsymbol{\delta}_{j}, \boldsymbol{y}\right\rangle=\left\langle\boldsymbol{P} \boldsymbol{\delta}_{j}, \boldsymbol{y}\right\rangle=\left\langle\boldsymbol{k}_{j}, \boldsymbol{y}\right\rangle$.

The third equality follows from the relation $\langle\boldsymbol{u}, \boldsymbol{A v}\rangle=\left\langle\boldsymbol{A}^{T} \boldsymbol{u}, \boldsymbol{v}\right\rangle$, which holds for any vectors, $\boldsymbol{u}$ and $\boldsymbol{v}$, and any square matrix $\boldsymbol{A}$ of the appropriate dimension. The fourth equality follows from (7), and the fifth is just the definition of $\boldsymbol{k}_{j}=\boldsymbol{P} \boldsymbol{\delta}_{j}$. Note that $\boldsymbol{k}_{j}$ is the $j^{\text {th }}$ row of $\boldsymbol{P}$ divided by $\Delta t$.

From (8) we can immediately calculate the standard deviation of the $R$-error

$$
\hat{s}_{j}=\left\langle\boldsymbol{k}_{j}, \boldsymbol{y}\right\rangle=\left\langle\boldsymbol{k}_{j}, \boldsymbol{s}\right\rangle+\left\langle\boldsymbol{k}_{j}, \boldsymbol{e}\right\rangle=s_{j}+\Delta t \sum_{i=1}^{N} k_{j i} e_{i} .
$$

The last term is the $R$-error. Since the $e_{i}$ are independent random variables with standard deviation $\sigma_{e}$, the variance of $\hat{s}_{j}, \sigma_{j}^{2}$, is given by

$\sigma_{j}^{2}=\Delta t^{2} \sigma_{e}^{2} \sum_{i=1}^{N} k_{j i}^{2}=\Delta t \sigma_{e}^{2}\left\|\boldsymbol{k}_{j}\right\|^{2}$, or $\sigma_{j}=\sqrt{\Delta t} \sigma_{e}\left\|\boldsymbol{k}_{j}\right\|$.

We are also interested in estimating the derivative of the signal and its variance as a function of time. The derivative of the signal is estimated by first performing the spline fit, as described previously, and then formally differentiating the result. Routines for performing the differentiation are available in the references. An approximation is used to estimate the variance of the derivative. If the sampling rate is high compared to the 
knot spacing (e.g., the time between samples is less than one fifth of the time between knots), then the derivative at the $j^{\text {th }}$ point is well approximated by

$\hat{s}_{j}^{\prime} \cong\left(\hat{s}_{j+1}-\hat{s}_{j-1}\right) /(2 \Delta t)=\left\langle\boldsymbol{\delta}_{j}^{1}, \hat{s}\right\rangle$, where

$\delta_{j k}^{1}=\left\{\begin{array}{c}1 /(2 \Delta t) \text { if } k=j+1 \\ -1 /(2 \Delta t) \text { if } k=j-1 \\ 0 \text { otherwise. }\end{array}\right.$

Here, $\hat{s}_{j}^{\prime}$ denotes the values of the derivative at the time corresponding to the $j^{\text {th }}$ sample. Following the same steps that lead to $(8)$ and (10) results in

$\sigma^{\prime}=\sqrt{\Delta t} \sigma_{e}\left\|\boldsymbol{k}_{j}^{\prime}\right\|$, with $\boldsymbol{k}_{j}^{\prime}=\boldsymbol{P} \boldsymbol{\delta}^{\prime}$.

In this section it was shown that the estimate of signal and its derivative and the variance of either as a function of time can be calculated using least-squares spline fitting routines that are readily available. The following sections show how to estimate the $F$-error as a function of time.

\section{INTUITIVE BASIS FOR THE $F$-ERROR ESTIMATE}

The $F$-error is estimated by comparing two different spline fits to the data. For any knot sequence, $K$, define an alternate knot sequence, $K^{\prime}$, as follows: The end points of $K^{\prime}$ are the same as those of $K$. In addition, a knot appears in $K^{\prime}$ at the midpoint between any two successive knots in $K$. Figure 1 shows the third derivative of the signal being fit as the smooth curve. Since the signal is assumed to have four derivatives, the third derivative is continuous. The third derivative of a cubic spline is constant between the knots and has jumps at the knots. The step function with solid lines represents the third derivative of the spline fit with the knot sequence, $K$. The step function with the dashed lines represents the third derivative of the spline fit with the knot sequence, $K^{\prime}$. The third derivative of the error for either fit is the difference between the step function and the curve.

It can be seen from the figure that the error in the third derivative is approximately a sawtooth function with jumps at the knots. The magnitude of the error is proportional to the fourth derivative of the signal (the slope of the third derivative). It can also be seen that the error from the alternate knot sequence is generally of the opposite sign of that form the base knot sequence. The two errors are approximately 90 degrees out of phase with each other. Integrating these sawtooth functions three times to obtain the error in the function values (or two times to get the error in the derivative) converts them to approximate sinusoids but maintains their approximately equal amplitude and their 90-degree phase difference. Neither of these two errors can be calculated, because the signal is unknown. However, the difference between the two errors can be calculated, because it is the difference between the two fitted signals. The difference between two sinewaves with the same amplitude and frequency and a 90-degree phase shift is a sinewave with an amplitude of 1.414 times the amplitude of the original sinewaves. Thus, examining the difference between the two spline fits may give a good indication of the F-error.

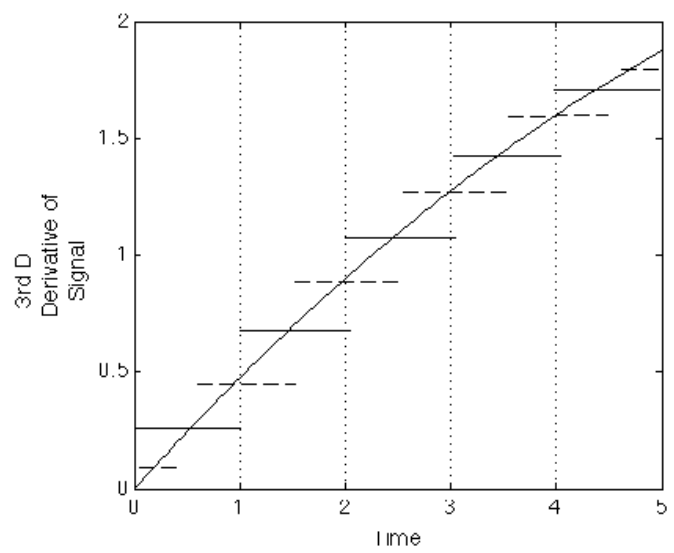

Fig. 1. The $3^{\text {rd }}$ derivative of a signal (smooth curve) along with the $3^{\text {rd }}$ derivative of two cubic spline approximations. The solid lines are for the knot sequence $K$ and the dashed lines are for the knot sequence $K$.

\section{THE $F$-ERROR ESTIMATE AND ITS MONTE CARLO EVALUATION}

The argument of the last section was based on the fourth derivative of the signal being nearly constant during each knot interval. The aim of this section is to quantitatively evaluate the situation of a non-constant fourth derivative and to construct a precise algorithm for estimating the $F$-error. In this section, we slightly modify the notation and use the sample time, $t$, as the independent variable rather than the sample index, $j$. Thus, in place of $s_{j}$ we will use $s(t)$ with $t$ being the time value corresponding to the $j^{\text {th }}$ sample. The quantities to be estimated are

$$
\begin{aligned}
& e_{t}=\sqrt{\mathrm{E}\left[(\hat{s}(t)-s(t))^{2}\right]} \quad \text { and } \\
& e_{t}^{\prime}=\sqrt{\mathrm{E}\left[\left(\hat{s}^{\prime}(t)-s^{\prime}(t)\right)^{2}\right]}
\end{aligned}
$$

Here $\mathrm{E}$ is the expectation operator, and the primes denote the derivative with respect to time. The approach consists of the following steps:

1. Construct the alternate knot sequence $K^{\prime}$ as described previously.

2. Calculate the alternate signal estimate, $\hat{s}_{K^{\prime}}(t)$, by calculating the vector $\hat{\boldsymbol{s}}_{K^{\prime}}=\boldsymbol{P}_{K^{\prime}} \boldsymbol{y}$. 
3. Calculate $\left[\begin{array}{l}\lambda(t)=\max \left\{\left|\hat{s}_{k^{\prime}}\left(t^{\prime}\right)-\hat{s}_{k}\left(t^{\prime}\right)\right|:\right. \\ \left.\text { for } t-\Delta_{k} / 2 \leq t^{\prime} \leq t+\Delta_{k} / 2\right\}\end{array}\right]$, where

$\Delta_{k}$ is the largest of the two mesh sizes (from $K$ and $K^{\prime}$ ) for the interval between two knots that contains $t$.

4. Let $e_{t}=0.5 \lambda(t)$.

The steps above give $e_{t}$. To obtain $e_{t}^{\prime}$, replace the function values with the derivatives in Step 3 and change the constant in Step 4 from 0.5 to 0.4. The values for these constants were obtained from the Monte Carlo simulations that follow. Note that (e.g. from Figure 1) the error goes through one period in an interval the length of the mesh size. Thus, Step 3 gives the maximum error over the one period nearest to the time of interest.

This method for estimating the $F$-error was tested on over 100000 signals using Monte Carlo simulations. The simulations were done with uniform knot spacing. It can easily be shown that the results, as presented here, are independent of the knot spacing. The filtering approach has no error for signals that are polynomials of degree three. Thus, by the Peano kernel theorem ([10] page 43 and [11] page 25), the approximation error depends only on the fourth derivative of the signal and depends linearly on it. The simulations were performed for signals whose fourth derivative was a Gaussian noise process with $\mathrm{rms}$ value of one and a power spectral density of the form

$$
S(\omega)=\frac{\tau}{\pi} \frac{1}{1+(\omega \tau)^{2}} .
$$

The corresponding autocorrelation function is

$$
R(t)=\exp \left(-\frac{|t|}{\tau}\right)
$$

The value of the correlation time, $\tau$, was varied between one-tenth of the mesh size and ten times the mesh size.

The simulations were performed with a sampling frequency of ten (i.e. ten samples per knot interval). A normally distributed random sequence was generated with the MATLAB random number generator. This sequence was filtered with a one-pole filter of the selected time constant. This gives a sequence with the power spectrum and autocorrelation function given above. This sequence was then numerically integrated four times to give a sample function.

For each sample function generated, the least-squares fit was performed, and the errors, $e(t)=\hat{s}(t)-s(t)$ and $e^{\prime}(t)=\hat{s}^{\prime}(t)-s^{\prime}(t)$ were calculated for $t$ at the knots and $t$ halfway between the knots. The error estimates, given by our proposed procedure, were also calculated at each of these times. The ratio of the actual error to the estimated standard deviation was saved in a histogram (one histogram for each value of $\tau$.) Ideally, the $r m s$ value of each of these histograms would be one. The actual values are shown in Figure 2 for signal errors and in Figure 3 for derivative errors.

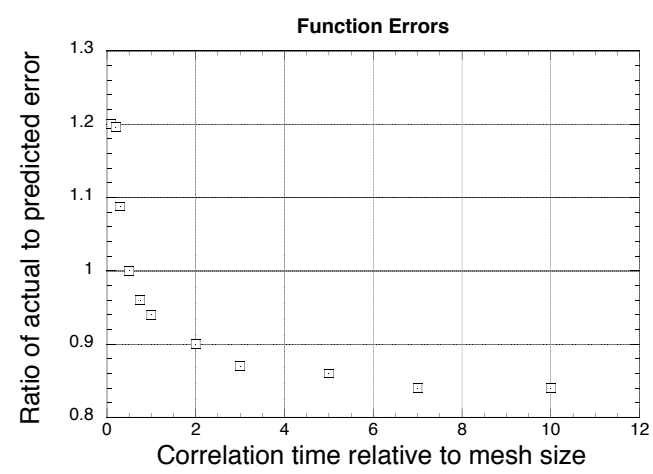

Fig. 2. The ratio of the actual rms error from (13) to the predicted rms error from Step 4 of the procedure of this section.

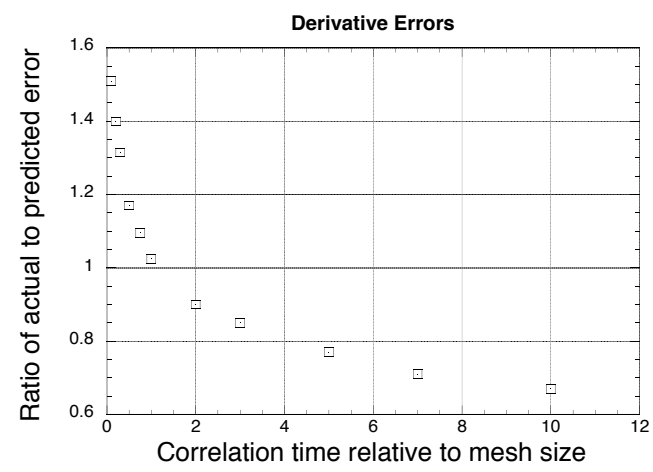

Fig. 3. The ratio of the actual rms error of the derivative from (13) to the predicted rms error from Step 4 of the procedure of this section modified for estimating the derivative error.

The constants in Step 4 of the estimation procedure were chosen to make the plots more or less symmetric about unity, and they apply to correlation times of one-half to three-quarters of the mesh size. Slightly smaller or larger constants could be used depending on the particular situation. The correlation time varies by a factor of 100 in Figures 2 and 3 while the function errors vary from $20 \%$ higher than the estimate for very short correlation times (rapidly varying fourth derivative) to $17 \%$ smaller for very long correlation times (slowly varying fourth derivative). For the derivative the pattern is the same but larger. The true error ranges from $50 \%$ above the estimate to $35 \%$ below the estimate.

\section{EXAMPLE}

The example presented in this section demonstrates the method applied to a signal that is much different than those used for the Monte Carlo simulations. The signal is of the form 


$$
s(t)=\left\{\begin{array}{c}
0 \text { for } t \leq 0 \\
\operatorname{erfc}\left(\sqrt{\frac{\tau}{t}}\right) \text { for } t>0
\end{array}\right.
$$

The derivative is given by

$$
s^{\prime}(t)=\sqrt{\frac{\tau}{\pi}} \frac{1}{t^{3 / 2}} e^{-\frac{\tau}{t}}
$$

These correspond to the step response and impulse response of a skin-effect limited coaxial cable. The step response rises very rapidly from $s(t)=0$ to $s(t)=0.5$, then very slowly from $s(t)=0.5$ to $s(t)=1.0$. The value used for $\tau$ is $0.2 \mathrm{~ns}$, which corresponds to about $30 \mathrm{~m}$ of RG-58 cable. The simulated sampling rate is $40 \mathrm{GSa} / \mathrm{s}$, a typical sampling rate for a modern high-speed digital oscilloscope. The signal was sampled for $1 \mu \mathrm{s}$, or 40000 samples. The mesh size, or knot spacing, varied from $0.1 \mathrm{~ns}$ near $t=0$ to $800 \mathrm{~ns}$ at the end of the record. This change in knot spacing by a factor of 8000 is very different from the uniform sampling used for the Monte Carlo simulations, but the results will show that the estimation procedure for $F$-errors still works remarkably well.

The error estimates were calculated at the knots and at the midpoints between the knots, so the knot locations can be determined form the error bar locations. The error bars in all figures are two standard deviations, twice the values given in the procedure of Section V. Two standard deviations corresponds to a $95 \%$ confidence level for errors if they have a Gaussian distribution.

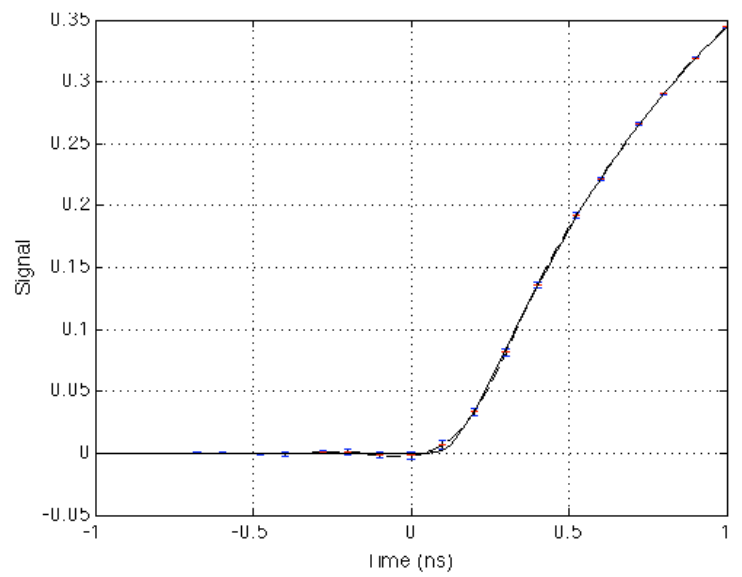

Fig. 4. The signal (given by (16), solid line), the spline fit (dashed line), and the 2-standard-deviaation error bars.

Figure 4 shows the early part of the signal (solid line), the fitted signal (dashed line), and the error bars. Figure 5 shows the error as a function of time along with the error bars -the agreement is very good. Figures 6 shows the same thing as figure 5 at later time.

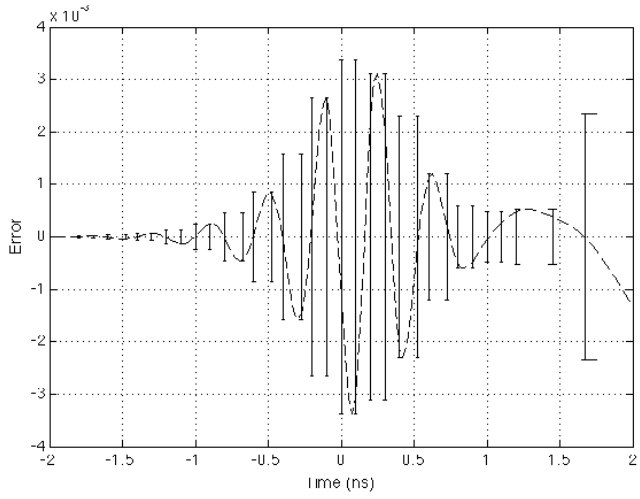

Fig 5. The difference between the input signal and the fitted signal of Figure 2 with the 2 -standard-deviation error bars. The full scale on the vertical axis is $4 \times 10^{-3}$.

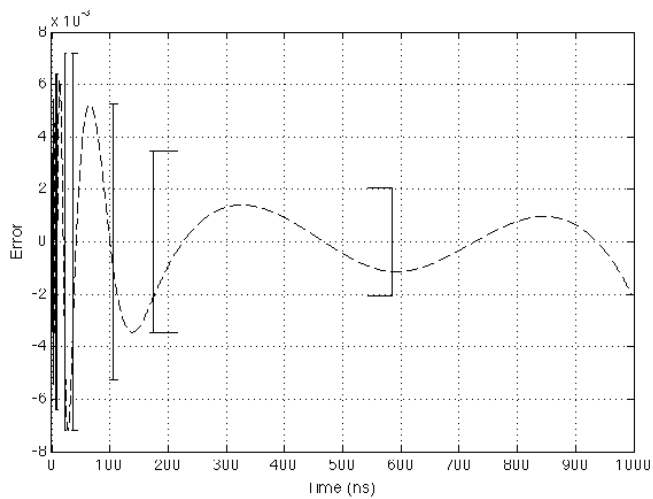

Fig. 6. The same as Figure 5 plotted for larger times. The full scale on the vertical axis is $8 \times 10^{-3}$.

Similar results were obtained for estimating the derivative. They will be shown in a paper with less stringent length restrictions.

\section{EFFECT OF NOISE ON $F$-ERROR ESTIMATE}

The analysis so far has compared the actual $F$-error with the $F$-error estimate obtained from noiseless data. In real applications, the $F$-error estimate is based on noisy data. This section contains an analysis of the effect of the noise on the estimate. For any particular time, $t$, the $F$-error estimate at time $t$ is given by

$e_{f}=0.5\left|\hat{s}_{K}\left(t_{0}\right)-\hat{s}_{K^{\prime}}\left(t_{0}\right)\right|=0.5\left|\left\langle\boldsymbol{k}_{t_{g}}, \boldsymbol{y}\right\rangle-\left\langle\boldsymbol{k}_{t_{0}}^{\prime}, y\right\rangle\right|=0.5\left|\left\langle\Delta \boldsymbol{k}_{t_{t^{\prime}}}, \boldsymbol{y}\right\rangle\right|$

where $t_{0}$ is the time at which the expression in Step 3 of Section $\mathrm{V}$ attains its maximum, $\boldsymbol{k}$ and $\boldsymbol{k}^{\prime}$ are the vectors from (8) for the knot sequences $K$ and $K^{\prime}$, and $\Delta \boldsymbol{k}=\boldsymbol{k}-\boldsymbol{k}^{\prime}$. The subscript $j$ from (8) has been replaced in (18) with its corresponding time. This gives

$e_{f}=0.5\left|\left\langle\Delta \boldsymbol{k}_{t_{0}}, s\right\rangle+\left\langle\Delta \boldsymbol{k}_{t_{0}}, \boldsymbol{e}\right\rangle\right|$.

The first term inside of the absolute value sign corresponds to the $F$-error estimate studied previously. 
The second term is a random error in the $F$-error estimate. Its standard deviation is proportional to $\left\|\Delta \boldsymbol{k}_{t_{0}}\right\|$, and the $R$ error, $e_{r}$, has a standard deviation proportional to $\left\|\boldsymbol{k}_{t}\right\|$ with the same proportionality constant. The ratio of the two errors is proportional to the ratio of the norms of these two vectors. For uniform knot spacing the vectors and their norms can be calculated. Since the ratio of the two norms is independent of the knot spacing (because both vectors follow the same scaling rules) it is sufficient to evaluate the norms for unit knot spacing. The result is shown in Figure 7. The horizontal axis is the subscript on the vector relative to the nearest knot. The vertical axis is 100 times the norm. We can see that $\|\Delta \boldsymbol{k}\| /\|\boldsymbol{k}\| \cong 0.5$, so the uncertainty in the $F$-error estimate is about 0.25 times the standard deviation of the $R$-error (because of the factor of $0.5 \mathrm{in})$. This means that the total error estimate is affected very little by the noise effect on the $F$-error estimate.

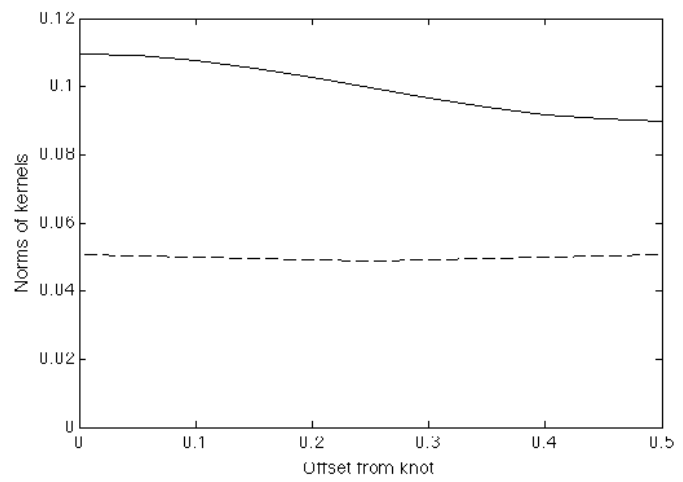

Fig. 7. The norm of the vector $\boldsymbol{k}$ of (18) (solid line) and the norm of $\Delta \boldsymbol{k}$ (solid line). The independent variable is the distance of the evaluation point from the nearest knot.

The entire argument can be repeated for estimating the derivative of the signal. For this we replace the $\boldsymbol{k}^{\prime}$ s in (18)

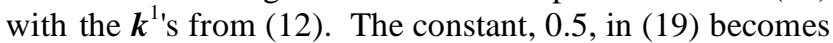
0.4 for the derivative estimation. In this case (there is no room for a figure), the value of $\left\|k^{1}\right\|$ varies by a factor of 1.74 , the value being lowest at the knot location and highest midway between the knots. If we use the value of $\left\|\boldsymbol{k}^{1}\right\|$ at the center, $t=0.25$, we get $\left\|\Delta \boldsymbol{k}^{1}\right\| /\left\|\boldsymbol{k}^{1}\right\| \cong 0.88$. Multiplying this by 0.4 (the analogous constant to the 0.5 in (19)), yields a ratio of the uncertainty in the $F$-error of about 0.35 times the standard deviation of the $R$-error.

\section{COMPARISON WITH SOME OTHER WORK}

The study of least-squares fitting with splines to reduce noise in measured data appears in Chapter XIV of [6] and in [9]. The idea of varying the knot density to match the local smoothness of the function being approximated is studied in Chapter XII of [6]. However, these studies use a priori knowledge about the unknown function rather than the data itself. The determining of good knot sequences based solely on the data was studied in [12] and [13]. However, none of the previously published work produces error estimates for the $F$-error.

\section{CONCLUSIONS}

This paper has shown how to estimate the error when fitting noisy data with spline functions. The error is divided into two sources: the $R$-error, which depends on the noise and increases with decreasing mesh size, and the $F$-error, which depends only on the signal and decreases with decreasing mesh size. The estimation of both errors was demonstrated. The $R$-error estimation requires knowledge of the statistics of the noise and uses wellknown methods. The $F$-error estimation requires no prior knowledge of the signal except that it has four derivatives. It is calculated from the difference between two different spline fits to the data and was illustrated with Monte Carlo Simulations and with an example.

\section{REFERENCES}

[1] Weiner, N., Time Series, M. I. T. Press, 1949

[2] Kolmogorov, A, "Interpolation und extrapolation von stationären zufälligen folgen, "Bulletin de l'académie des sciences de U.R.S.S., Ser. Math., pp. 3-14, 1941.

[3] Kosulajeff, P., "Sur les problèmes d'interpolation et d'extraplation des suites stationnaires", Comptes rendus de l'académie des sciences des U.R.S.S., vol. 30, pp. 13-17, 1941.

[4] Gauss, K., Theory of the Combination of Observations Least Subject to Errors, SIAM, 1995.

[5] Kay, S., Fundamentals of Statistical Signal Processing - Estimation Theory, Prentice Hall, 1993.

[6] de Boor, C., A Practical Guide to Splines - Revised Edition, Springer, 2001.

[7] Spline Toolbox, The Mathworks.

[8] Unser, M., A. Aldroubi, and M. Eden, "Polynomial spline signal approximations: filter design and asymptotic equivalence with Shannon's sampling theorem, "IEEE Trans. Info. Theory, vol. 38, pp. 95-103, Feb. 1992

[9] Unser, M., A. Aldroubi, and M. Eden, "B-spline signal processing: part I - theory,"IEEE Trans. Signal Processing, vol. 41, pp. 821832, Feb. 1993.

[10] Ralston, A. and P. Rabinowitz, First Course in Numerical Analysis, McGraw-Hill, 1978.

[11] Sard, A., Linear Approximation, American Mathematical Society, 1963.

[12] He, X., L. Shen and Z. Shen, "A data-adaptive knot selection scheme for fitting splines," Signal Proc. Letters, vol. 8, No. 5, pp. 137-139, May 2001.

[13] Ainsleigh, P. and C. Chui, "Simultaneous wavelet and spline smoothing of noisy data," Acoustics, Speech and Signal Processing, 1993 IEEE International Conference on, vol. 3, pp. 197-200, Apr. 1993.

This manuscript, DOE/NV/25946--132, has been authored by National Security Technologies, LLC, under Contract No. DE-AC5206NA25946 with the U.S. Department of Energy. The United States Government retains and the publisher, by accepting the article for publication, acknowledges that the United States Government retains a nonexclusive, paid-up, irrevocable, worldwide license to publish or reproduce the published form of this manuscript, or allow others to do so, for United States Government purposes. 